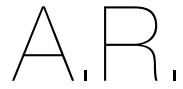

ARTIGO DE REVISÃO

1 Escola Superior de

Tecnologia da Saúde de

Coimbra,

Rua 5 de Outubro,

3046-854 Coimbra,

Portugal

"Endereço para correspondência:

Daniela Silva Martins

Rua Principal, n. ${ }^{\circ} 6$

Anços, Portugal

daniela_fm16@hotmail.com

Histórico do artigo:

Recebido a 9 de setembro de 2019 Aceite a 30 de setembro de 2019

\title{
ALIMENTAÇÃO VEGETARIANA NA CRIANÇA E NO ADOLESCENTE
}

Daniela Silva Martins ${ }^{1 *}$; Ana Faria'; Helena Loureiro

\section{RESUMO}

Os comportamentos alimentares têm vindo a alterar-se ao longo dos anos, tendo-se verificado um maior interesse pelo padrão alimentar vegetariano, por parte de adultos mas também de crianças e adolescentes.

O objetivo deste artigo é identificar os motivos que provocam uma adesão ao padrão alimentar vegetariano e identificar os efeitos sobre a saúde deste padrão alimentar nas crianças e adolescentes.

Alguns dos motivos que podem levar as crianças e adolescentes a adotarem por um padrão alimentar vegetariano podem ser por questões de saúde, ambientais, religiosas ou éticas.

O padrão alimentar em questão apresenta alguns benefícios para a saúde, nomeadamente na prevenção de doenças como a obesidade, doenças cardiovasculares, diabetes tipo 2 e cancro.

Por fim, o padrão alimentar vegetariano, se for corretamente planeado, pode fornecer as necessidades nutricionais de crianças $e$ adolescentes. Contudo, se não for bem planeado pode originar défices nutricionais, comprometendo o crescimento e desenvolvimento adequados nestas faixas etárias.

\section{PALAVRAS-CHAVE}

Adolescentes, Crianças, Dieta vegetariana, Saúde

\section{ABSTRACT}

Eating behaviors have been changing over the years and there has been a greater interest in the vegetarian dietary pattern by adults but also by children and adolescents.

The aim of this paper is to identify the reasons for adhering to the vegetarian dietary pattern and to identify the health effects of this dietary pattern on children and adolescents.

Some of the reasons that may lead children and adolescents to adopt a vegetarian dietary pattern may be for health, environmental, religious or ethical reasons.

The dietary pattern in question has some health benefits including the prevention of diseases such as obesity, cardiovascular disease, type 2 diabetes and cancer.

Finally, the vegetarian dietary pattern, if properly planned, can provide the nutritional needs of children and adolescents. However, if not well planned can lead to nutritional deficits, compromising proper growth and development in these age groups.

KEYWORDS

Adolescents, Children, Vegetarian diet, Health

\section{INTRODUÇÃO}

O padrão alimentar vegetariano, é um padrão de consumo alimentar, também designado por "alimentação vegetariana" ou "dieta vegetariana" (1) que tem vindo a ganhar popularidade em todo o mundo (2), bem como em Portugal (3). Apesar de não existirem muitos dados (4), supõe-se que esteja a aumentar a prevalência de bebés, crianças e adolescentes que praticam este tipo de alimentação $(5,6)$.

De acordo com a Sociedade Vegetariana Brasileira (7) "é considerado vegetariano todo aquele que exclui da sua alimentação todos os tipos de carne, aves, peixes e seus derivados, podendo ou não utilizar laticínios ou ovos."
O padrão alimentar vegetariano pode classificar-se em ovolactovegetariano (exclui a carne e o pescado e permite ovos e laticínios) (5, 7-9), lactovegetariano (exclui a carne, pescado e ovos e permite os laticínios) (5, 7-9), ovovegetariano (exclui carne, pescado e laticínios e permite o consumo de ovos) (5, 7-9), vegetariano estrito (exclui todos os alimentos de origem animal (incluindo, por ex.: mel, gelatina, banha, insetos, albumina, caseína, corante E120)) e vegan (exclui todos os alimentos de origem animal e todos os produtos que os contenham, nomeadamente vestuário, adornos, produtos testados em animais e condena a utilização de animais como forma de entretenimento) $(1,5,7)$. 
Os principais constituintes de um padrão alimentar vegetariano são a fruta, os hortícolas, os cereais e tubérculos, as leguminosas, os frutos gordos e as sementes, de preferência produzidos localmente, da época e minimamente processados (5).

A Academia Americana de Nutrição e Dietética (8) considera que "dietas vegetarianas adequadamente planeadas, incluindo as vegan, são saudáveis, nutricionalmente adequadas e podem fornecer benefícios à saúde na prevenção e tratamento de certas doenças. São apropriadas para todas as etapas do ciclo de vida, incluindo gravidez, lactação, infância, adolescência, idade adulta e para atletas." Contudo, a Associação Alemã de Nutrição (4) não recomenda uma dieta vegan na infância e adolescência devido ao maior risco de défices nutricionais.

Motivos que levam à adesão a um padrão alimentar vegetariano Desde a Antiguidade Clássica que se conhecem e seguem padrões alimentares vegetarianos, essencialmente por questões filosóficas, religiosas e de saúde (1). Os motivos que levam à adesão a um padrão alimentar vegetariano são diversos nomeadamente:

1. Éticos e filosóficos - Quando os indivíduos decidem evitar comer carne por acreditarem que o ato de a comerem é errado, por terem a noção de que os animais são seres sencientes. Os animais não deviam ser criados e abatidos com o propósito de serem utilizados para fins alimentares, de cosmética e vestuário $(7,10)$.

2. Saúde - Uma alimentação vegetariana apresenta alguns benefícios para a saúde, nomeadamente na diminuição do risco de diabetes tipo $2(1,6,8,9)$, doenças cardiovasculares $(1,6,9)$, hipertensão (1, $6,8)$, hiperlipidemia $(1,6)$, obesidade $(1,8,11,12)$, cancro da mama (9), cancro do cólon (9), doenças da vesícula biliar (9), aumento da longevidade (1) e apresentam um perfil mais favorável de adipocinas anti-inflamatórias (6).

3. Ambientais - De acordo com a Sociedade de Educação e Comportamento Nutricional Americana (11) as tendências ambientais atuais (ex.: alterações climáticas, perda de biodiversidade, escassez de água) ameaçam a segurança alimentar a longo prazo, e em parte, são causadas pelas escolhas alimentares e práticas agrícolas atuais. O impacto ambiental pode ser diminuído reduzindo o consumo de alimentos de origem animal e aumentando o consumo de alimentos de origem vegetal (11).

A produção dos alimentos que integram a alimentação vegetariana utiliza menos recursos de água, combustíveis fósseis, pesticidas e fertilizantes, comparada com uma alimentação omnívora (8).

De acordo com a Organização para a Alimentação e Agricultura (13) "Dietas sustentáveis são aquelas com baixo impacto ambiental que contribuem para a segurança alimentar e nutricional, para a saúde e vida das gerações presentes e futuras. As dietas sustentáveis protegem e respeitam a biodiversidade e os ecossistemas, são culturalmente aceitáveis, disponíveis, economicamente justas e acessíveis, nutricionalmente adequadas, seguras e saudáveis, enquanto contribuem para otimizar os recursos naturais e humanos". Deste modo podemos considerar que o padrão alimentar vegetariano apresenta efeitos favoráveis na sustentabilidade ambiental (8).

4. Familiares - Por vezes as crianças e adolescentes, assim como a população adulta são influenciadas a adotar este tipo de alimentação por familiares (7) ou influenciadores nas redes sociais.

5. Religiosos - Algumas religiões como a Igreja Adventista do Sétimo Dia, o Espiritismo, o Hinduísmo, o Budismo, entre outras, recomendam a adoção de um padrão alimentar vegetariano (7).

6. Sociopolíticos - Quando os indivíduos decidem evitar a carne por questões de segurança alimentar e de justiça social (10), no sentido de haver alimento disponível para todas as pessoas.

7. Paladar - Pode haver recusa do consumo de carne por não aceitação do paladar (7). De acordo com Ramalho C. e col. (14), no seu estudo sobre neofobia alimentar em crianças dos 6 aos 12 anos, os novos alimentos com menor intenção de serem experimentados eram sobretudo de origem animal.

\section{Benefícios para a saúde de um padrão alimentar vegetariano}

A evidência científica sobre os benefícios do padrão alimentar vegetariano tem vindo a aumentar nos últimos anos, nomeadamente na prevenção de algumas doenças crónicas que afetam a nossa sociedade (3). Um padrão alimentar vegetariano adequado na infância pode reduzir os riscos de algumas doenças crónicas na idade adulta (5), nomeadamente:

1. Obesidade - A obesidade infantil é um problema de saúde pública do século XXI (15), que tem vindo a aumentar em áreas urbanizadas de países em desenvolvimento (12), e está associada a uma maior morte prematura e incapacidade na idade adulta $(12,15)$.

As principais consequências do sobrepeso e obesidade infantil para a saúde, que geralmente aparecem na idade adulta, são doenças cardiovasculares, diabetes, distúrbios músculo-esqueléticos e certos tipos de cancro (endométrio, mama e cólon) (15).

Alguns estudos referem que as dietas vegetarianas estão associadas a um menor Índice de Massa Corporal (IMC) e menor prevalência de obesidade em adultos e crianças $(8,12)$. Em comparação com crianças não vegetarianas, as vegetarianas são mais magras e a diferença de IMC aumenta durante a adolescência (12). Um estudo realizado em crianças e adolescentes adventistas com idades entre os 7 e os 18 anos, classificadas como vegetarianas, observou que as crianças vegetarianas eram mais altas que os colegas omnívoros (12). Contudo, numa pesquisa longitudinal realizada em crianças e adolescentes adventistas e omnívoros entre os 6 e os 18 anos, observou-se que as meninas pré-púberes adventistas eram mais baixas, e que, nas meninas omnívoras o início do surto de crescimento puberal ocorreu um ano antes, o que sugere que as meninas adventistas vegetarianas apresentam um atraso no início da maturação puberal, o que pode reduzir o risco de desenvolver cancro da mama (12). Um estudo realizado em adolescentes adventistas que frequentavam o ensino secundário mostrou que os estudantes com uma alimentação predominantemente vegetariana tinham menores valores de IMC, circunferência da cintura, relação colesterol total e colesterol HDL e concentração do colesterol LDL, em comparação com os estudantes omnívoros (12). As dietas à base de plantas apresentam uma baixa densidade energética $(4,12)$, e um elevado teor de hidratos de carbono complexos, fibra e água que aumentam a saciedade e o gasto energético em repouso (12).

Sabaté e Wen (12) relataram que os alimentos de origem animal (carne, laticínios e ovos) estão associados a um aumento do risco de excesso de peso, enquanto os alimentos de origem vegetal são protetores (cereais, hortícolas, nozes) ou não apresentam associação (fruta, hortícolas e produtos de proteína vegetal). Os autores descreveram que as diferenças observadas no IMC entre vegetarianos e não vegetarianos podem ser explicadas pelo consumo de carne (rica em ácidos gordos saturados e com maior densidade calórica), por fatores de estilo de vida (ex.: tabagismo, atividade física e nível de educação) e por uma maior ingestão e/ou variedade de hortofrutícolas. Deste modo, um padrão alimentar vegetariano pode ser importante na prevenção primária de excesso de peso e obesidade (12). A ingestão alimentar elevada de proteína, principalmente de origem animal, está positivamente associada ao IMC (12) e é um fator que predispõe para o excesso de peso e obesidade em crianças $(6,12)$. 
2. Estado de inflamação - Ambroszkiewicz e col. (6) descreveram que um padrão alimentar ovolactovegetariano, quando bem planeado pode afetar beneficamente o perfil de adipocinas e pode ter um efeito protetor sobre o estado inflamatório em crianças pré-púberes com idades entre os 5 e os 10 anos, comparativamente a uma dieta omnívora tradicional. As adipocinas secretadas pelo tecido adiposo, estão envolvidas de maneira autócrina e parácrina na regulação do gasto de energia, na sensibilidade à insulina, no metabolismo de glicose e lipídico, na função endotelial e no estado inflamatório (6). Entre as adipocinas, a leptina, a resistina e a visfatina, foram descritas como marcadores positivamente relacionados com o peso corporal, a massa gorda, a resistência à insulina e propriedades pró-inflamatórias (6). No estudo realizado por Ambroszkiewicz e col. (6) observou-se que os níveis da adipocina leptina eram menores nas crianças vegetarianas em comparação com as omnívoras. A desregulação da síntese de adipocinas pode alterar a homeostase e levar a condições patológicas (6). De acordo com alguns autores, os principais compostos bioativos associados às propriedades anti-inflamatórias e antioxidantes são os polifenóis, as vitaminas e os minerais que se obtém nos alimentos de origem vegetal (6). O padrão alimentar vegetariano e a dieta mediterrânea estão associados a um menor estado inflamatório, a níveis mais elevados de adiponectina, níveis mais baixos de leptina e níveis inalterados de resistina (6).

3. Doenças cardiovasculares (hiperlipidemia, cardiopatia isquémica e hipertensão) - O consumo de um padrão alimentar vegetariano está associado a uma redução do risco de desenvolver doenças cardiovasculares (8), devido à melhoria de fatores de risco modificáveis, nomeadamente obesidade abdominal, pressão arterial (16), perfil lipídico, glicémia, diminuição da proteína $\mathrm{C}$ reativa, redução do stress oxidativo e redução da formação de placas ateroscleróticas (8). Alguns estudos verificaram uma redução da prevalência de hipertensão arterial sistémica em populações vegetarianas. Esta redução está relacionada com uma maior sensibilidade à insulina e um melhor estado antioxidante, que melhora a redução da aterogenicidade e preserva o óxido nítrico sintetizado pelo endotélio (7). Os indivíduos que praticam um padrão alimentar vegetariano apresentam um nível sérico mais elevado de diversos antioxidantes, atividade da superóxidodismutase, maior proteção contra a oxidação das lipoproteínas e maior estabilidade genómica. No entanto, os indivíduos vegetarianos que não fazem suplementação de vitamina B12, apresentam níveis maiores de homocisteína, o que aumenta a formação de radicais livres (7).

4. Diabetes tipo 2 - Os indivíduos com um padrão alimentar vegan ou ovolactovegetariano têm menor risco de desenvolver diabetes tipo 2 relativamente a indivíduos omnívoros. O consumo elevado de cereais integrais, hortícolas, fruta e sementes e o baixo consumo de cereais refinados, bebidas açucaradas e de carnes vermelhas ou processadas, reduz o risco de diabetes e melhora o controlo glicémico e o perfil lipídico em pessoas com diabetes (8).

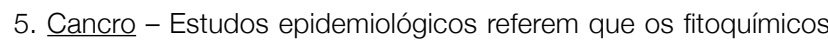
presentes nos alimentos de origem vegetal estão associados a um risco reduzido de certos tipos de cancro $(8,17)$. Alguns estudos verificaram uma menor prevalências de alguns tipos de cancro em indivíduos vegetarianos, tendo sido associados fatores como um menor valor de IMC, um melhor estado antioxidante e inflamatório e a um menor nível de insulina, encontrados em indivíduos vegetarianos (7). O consumo excessivo de carne aumenta o risco de cancro do cólon e reto (7).

6. Osteoporose - A elevada ingestão de produtos hortofrutícolas está associada a alguns fatores que promovem a saúde óssea. Contudo, a baixa ingestão de cálcio, vitaminas D e B12 e proteína pode comprometer a saúde óssea, principalmente no padrão alimentar vegan (8).

7. Menor exposição a antibióticos - Crianças com uma alimentação vegetariana estão menos expostas a antibióticos veterinários, encontrados em alimentos de origem animal (2), e que podem provocar resistência a antibióticos, causando doenças difíceis de tratar e aumentando a morbilidade e mortalidade (8).

\section{Riscos nutricionais associados a um padrão alimentar vegetariano}

Os nutrientes que apresentam um maior risco de carência nos praticantes deste padrão alimentar são a vitamina B12, a vitamina D, os ácidos gordos ómega 3, a proteína, o cálcio, o zinco, o ferro e o iodo $(2,4,5,18)$ devendo ser realizado um correto planeamento e uma monitorização adequada, de modo a colmatar as possíveis carências nutricionais, tendo em atenção a biodisponibilidade dos nutrientes $(2,18$, 19). Em alguns casos pode ser necessária a ingestão de suplementos e/ou de alimentos fortificados $(5,8,18)$.

Os bebés, as crianças e os adolescentes são mais vulneráveis e apresentam maior risco de inadequação nutricional do que os adultos, uma vez que apresentam maiores necessidades de energia e nutrientes durante o crescimento (4).

De acordo com alguns autores, a dieta vegan é o único padrão alimentar vegetariano que pode apresentar risco de nutrição inadequada, caso não seja bem planeado (9) e a dieta ovolactovegetariana é a que se associa a um menor risco no desenvolvimento e crescimento das crianças (19).

Durante a adolescência (8) podem desenvolver-se distúrbios alimentares como a bulimia (5), a anorexia nervosa (7) e a ortorexia (7), contudo não existe evidência de que o consumo de um padrão alimentar vegetariano aumente o risco de um distúrbio alimentar, embora adolescentes com distúrbios alimentares pré-existentes possam adotar este tipo de alimentação para limitar a ingestão de alimentos $(5,8)$

\section{ANÁLISE CRÍTICA}

Um padrão alimentar vegetariano saudável deve respeitar as regras básicas de uma alimentação saudável, nomeadamente o fornecimento de refeições completas, equilibradas e diversificadas; a ingestão adequada de água; e a redução do consumo de açúcar, sal e gordura saturada e trans $(1,3)$. Deve fornecer energia e nutrientes em quantidades adequadas para o correto funcionamento do organismo, permitindo um crescimento e desenvolvimento adequado de crianças e adolescentes $(2,5)$.

O padrão alimentar vegetariano, apresenta algumas características do padrão alimentar Mediterrânico, nomeadamente o consumo elevado de alimentos de origem vegetal e o consumo de produtos frescos, minimamente processados e produzidos localmente, respeitando a sazonalidade $(5,20-23)$. Ambos os padrões alimentares estão associados com uma maior longevidade, menor índice de inflamação (6), proteção contra doenças como o cancro, a diabetes tipo 2, a hipertensão arterial, a doença cardiovascular e a obesidade $(1,20)$. De acordo com dados preliminares da 5. ${ }^{a}$ fase do COSI Portugal (24), verificou-se uma diminuição de $8,3 \%$ na prevalência de excesso de peso infantil (37,9\% para 29,6\%) e de 3,3\% na prevalência de obesidade infantil (15,3\% para 12,0\%), entre 2008 e 2019, em crianças entre os 6 e os 8 anos. Contudo, os resultados do Inquérito Alimentar Nacional e de Atividade Física (25) revelaram que o grupo etário das crianças (3-9 anos) e dos adolescentes (10-17 anos) é onde se verifica um menor consumo de fruta e hortícolas. Deste modo, a implementação da opção vegetariana $(26,27)$ nas ementas escolares foi uma medida 
importante uma vez que num padrão alimentar vegetariano existe um maior consumo de alimentos de origem vegetal (5).

Por fim, o padrão alimentar vegetariano, se não for bem planeado pode ter consequências para a saúde, deste modo são necessárias escolhas alimentares adequadas e um estilo de vida saudável.

\section{CONCLUSÕES}

Relativamente aos motivos que levam a uma adesão a um padrão alimentar vegetariano por parte de crianças e adolescentes são necessários mais estudos nesta população.

Em relação aos riscos e benefícios de um padrão alimentar vegetariano, desde que bem planeado e monitorizado por um profissional de saúde qualificado, os vários tipos de dietas vegetarianas são adequadas nas crianças e adolescentes, apresentando vários benefícios para a saúde a curto e a longo prazo. Contudo, se não for bem planeado pode originar défices nutricionais, comprometendo o crescimento e desenvolvimento adequados das crianças e adolescentes.

Por fim, são necessários mais estudos realizados em crianças e adolescentes que pratiquem um padrão alimentar vegetariano em comparação com grupos de controlo que pratiquem uma alimentação omnívora para analisar os efeitos na saúde deste padrão alimentar.

\section{REFERÊNCIAS BIBLIOGRÁFICAS}

1. Silva S C G, et al. Linhas de orientação para uma alimentação vegetariana saudável. Programa Nacional para a Promoção da Alimentação Saudável. Direção-Geral da Saúde. 2015. Disponível em: http://nutrimento.pt/noticias/linhas-de-orientacao-parauma-alimentacao-vegetariana-saudavel/.

2. Baroni L, et al. Vegan Nutrition for Mothers and Children: Practical Tools for Healthcare Providers. Nutrients. 2019. 11, 5. Disponível em https://doi.org/10.3390/nu11010005. 3. Lobato L, Silva SCG, et al. Planeamento de refeições vegetarianas para crianças em restauração coletiva: Princípios Base. Programa Nacional para a Promoção da Alimentação Saudável. Direção-Geral da Saúde. 2016. Disponível em: http:// nutrimento.pt/noticias/planeamento-de-refeicoes-vegetarianas-para-criancas-emrestauracao-coletiva-principios-base/.

4. Schürmann S, Kersting M, \& Alexy U. Vegetarian diets in children: a systematic review. European Journal of Nutrition. 2017. 56(5), 1797-1817.Disponivel em:doi:10.1007/ s00394-017-1416-0.

5. Pinho J P, et al. Alimentação vegetariana em idade escolar. Programa Nacional para a Promoção da Alimentação Saudável. Direção-Geral da Saúde. 2016. Disponível em: http://nutrimento.pt/noticias/alimentacao-vegetariana-em-idade-escolar/.

6. Ambroszkiewicz J, et al. Anti-Inflammatory and Pro-Inflammatory Adipokine Profiles in Children on Vegetarian and Omnivorous Diets. Nutrients.2018.10, 1241. Disponível em: doi:10.3390/nu10091241.

7. Sociedade Vegetariana Brasileira. Guia alimentar de dietas vegetarianas para adultos. São Paulo. 2012. Disponível em: https://www.svb.org.br/publicacoes/livros. Acedido em 11/08/2019.

8. Melina V, Craig W, Levin S. Position of the Academy of Nutrition and Dietetics: Vegetarian Diets. Journal of the Academy of Nutrition and Dietetics: 2016;116(12):1970 1980. Disponivel em: https://doi.org/10.1016/j.jand.2016.09.025.

9. Mahan LK, Escott- Stump S. Krause: Alimentos, Nutrição e Dietoterapia $12^{\mathrm{a}}$ ed. São Paulo; 2010.

10. Naconecy C.. Ética \& Vegetarianismo. Sociedade Vegetariana Brasileira. 2015. Disponível em: https://www.svb.org.br/publicacoes/livros. Acedido em 11/08/2019.

11. Rose D. et al. Position of the Society for Nutrition Education and Behavior: The Importance of Including Environmental Sustainability in Dietary Guidance. Journal of Nutrition Education and Behavior. 2019. 51,1. Disponível em: https://doi.org/10.1016/j. jneb.2018.07.006.

12. Sabaté J, Wien M. Vegetarian diets and childhood obesity prevention. The American Journal of Clinical Nutrition. 2010. 91;1525S-1529S. Disponível em: https://doi. org/10.3945/ajen.2010.28701F.
13. Burlingame B., et al., Sustainable Diets and Biodiversity in Directions and Solutions for Policy, Research and Action. Food and Agriculture Organization. 2012.

14. Ramalho C, et al. Neofobia alimentar em crianças do $1 .{ }^{\circ}$ ciclo e seus cuidadores. Acta Portuguesa de Nutrição.2016.

15. Centro de estudos e Investigação em Dinâmicas Sociais e Saúde. About Childhood Obesity. 2016. Disponível em: http://www.ceidss.com/about-childhood-obesity/. Acedido em 27/08/2019.

16. Key TJ, et al. Health effects of vegetarian and vegan diets. Proceedings of the Nutrition Society. 2006. 65, 35-41. Disponível em: DOI:10.1079/PNS2005481.

17. Craig W J. \& Mangels A R. Position of the American Dietetic Association: vegetarian diets. J. Am. Diet. Assoc. 2009. 109, 1266-1282.

18. Agnoli C, et al. Position paper on vegetarian diets from the working group of the Italian Society of Human Nutrition. Nutrition, Metabolism \& Cardiovascular Diseases 2017. 27, 1037e1052. Disponível em: https://doi.org/10.1016/j.numecd.2017.10.020. 19. Pimentel D, Tomada I, Rêgo C. Alimentação vegetariana nos primeiros anos de vida: considerações e orientações. Acta Portuguesa de Nutrição. 2018; edição 14: 10-17. 20. In Brochura "Dieta Mediterrânica - um património civilizacional partilhado". 2013. ISBN 978-972-8103-74-3. Disponível em: http://www.dgs.pt/documentos-epublicacoes/dieta-mediterranica-um-patrimonio-civilizacional-partilhado.aspx.

21. Pinho I, et al. Guia Alimentar Mediterrânico: Relatório justificativo do seu desenvolvimento. Programa Nacional para a Promoção da Alimentação Saudável. Direção-Geral da Saúde. 2016. Disponível em: https://nutrimento.pt/noticias/guiaalimentar-mediterranico-relatorio-justificativo-do-seu-desenvolvimento/.

22. Fundación Dieta Meterránea. Dieta Mediterránea. Disponível em: https:// dietamediterranea.com/nutricion-saludable-ejercicio-fisico/. Acedido em 13/08/2019. 23. Serra-Majem, et al. Does the definition of the Mediterranean diet need to be updated? Public Health Nutrition. 2004, 7 (7): 927-929.

24. Instituto Ricardo Jorge. Childhood Obesity Surveillance Initiative - COSI PORTUGAL 2019. Disponível em: http://www.insa.min-saude.pt/cosi-portugal-2019-excessode-peso-e-obesidade-infantil-continuam-em-tendencia-decrescente/. Acedido em 27/08/2019.

25. Lopes C, Torres D, Oliveira A, et al. Inquérito Alimentar Nacional e de Atividade Física, IAN-AF 2015-2016: Relatório de resultados. Universidade do Porto, 2017. ISBN: 978-989-746-181-1. Disponível em: www.ian-af.up.pt.

26. Assembleia da República. Lei n. 11/2017. Diário da República, N. ${ }^{\circ} 75 / 2017$, Série I; 17 de abril de 2017.Disponivel em: https://dre.pt/pesquisa/-/search/106886578/ details/normal?q=Lei+n.\%C2\%BA\%2011\%2F2017\%2C\%20de+17+de+abril.

27. Lima, R.M. Orientações sobre ementas e refeitórios escolares. Ministério da Educação - Direção-Geral da Educação. 2018. Disponível em: https://www.dge.mec. pt/noticias/educacao-saude/orientacoes-sobre-ementas-e-refeitorios-escolares-2018. 